Complex Bragg grating writing using direct modulation of the optical fiber with flexural waves

Roberson A. Oliveira, Carlos A. F. Marques, Kevin Cook, John Canning, Rogério N. Nogueira, and Alexandre A. P. Pohl

Citation: Appl. Phys. Lett. 99, 161111 (2011); doi: 10.1063/1.3655328

View online: http://dx.doi.org/10.1063/1.3655328

View Table of Contents: http://aip.scitation.org/toc/apl/99/16

Published by the American Institute of Physics

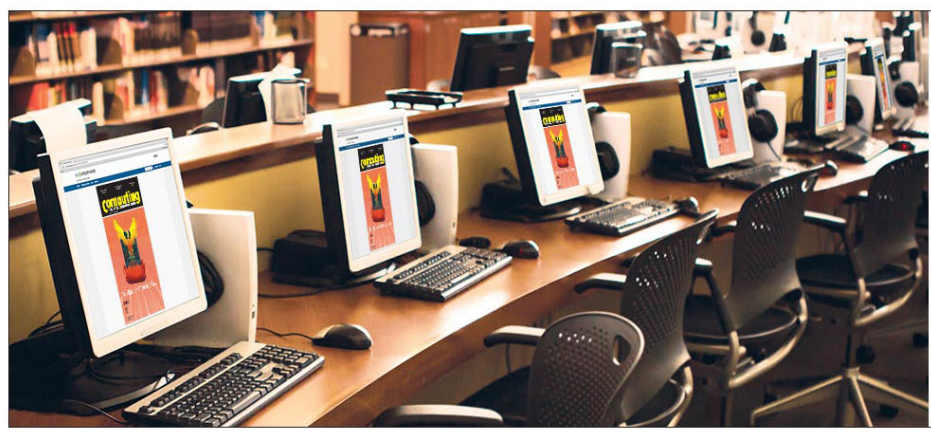
your fingertips... 


\title{
Complex Bragg grating writing using direct modulation of the optical fiber with flexural waves
}

\author{
Roberson A. Oliveira, ${ }^{1, a)}$ Carlos A. F. Marques, ${ }^{2}$ Kevin Cook, ${ }^{3}$ John Canning, ${ }^{3}$ \\ Rogério N. Nogueira, ${ }^{2}$ and Alexandre A. P. Pohl ${ }^{1}$ \\ ${ }^{1}$ Federal University of Technology - Paraná, Ave. Sete de Setembro, 3165, Curitiba 80.230-901, Paraná, \\ Brazil \\ ${ }^{2}$ Instituto de Telecomunicações, Pólo de Aveiro, Aveiro 3810-193, Portugal \\ ${ }^{3}$ Interdisciplinary Photonics Laboratories, 222 Madsen Building F09, The University of Sydney, Sydney, \\ New South Wales 2006, Australia
}

(Received 16 June 2011; accepted 3 October 2011; published online 19 October 2011)

\begin{abstract}
A flexural wave is applied to an optical fiber during the process of Bragg grating inscription using the direct writing method through a phase mask. Using this approach, we can dither the writing process to allow complex grating writing. Examples we demonstrate are tunable sampled gratings and phase-shifted gratings. (C) 2011 American Institute of Physics. [doi:10.1063/1.3655328]
\end{abstract}

The inscription of optical fiber Bragg gratings using the method of direct writing through a phase mask has been employed for almost two decades, allowing the efficient inscription of uniform and non-uniform Bragg gratings. ${ }^{1}$ This technique has made possible the development of a vast range of telecommunication and sensing devices that are based on, for example, sampled gratings ${ }^{2,3}$ and phase-shifted gratings. ${ }^{4}$ The range of applications of such grating structures can be extended through the use of the acousto-optic effect. ${ }^{5-8}$ However, the modulation caused by this effect disappears in the absence of the acoustic wave.

In this work, we investigate how to control the excitation of an acoustic flexural wave during the inscription of permanent Bragg grating structures. This mechanism was first demonstrated for the inscription of permanent sampled gratings using longitudinal acoustical waves. ${ }^{9}$ Here, we expand to the case where flexural waves are excited leading to dithering of the fiber.

When longitudinal waves are launched in the fiber, the strain field causes alterations in the impermeability tensor, which is converted to changes in the effective refractive index, described by

$$
\Delta\left(\frac{1}{n_{e f f}^{2}}\right)_{i}=\sum_{j=1}^{6} p_{i j} \varepsilon_{j}, \quad \mathrm{i}=1,2,3, \ldots, 6
$$

where $p_{i j}$ are the components of the elasto-optic tensor and $\varepsilon_{j}$ is the strain sensor. Once the acoustic wave modifies the impermeability tensor periodically, compression and rarefaction fields are generated, which modify the photoelastic coefficient. ${ }^{9}$ Thus, by adjusting the stress profile along the grating, the UV-induced index change is altered by the corresponding stress profile. On the other hand, when a flexural wave excites the fiber during inscription, periodic bending fields are generated. ${ }^{10}$ This bending causes the misplacing of the UV interference pattern (generated by the inscription light) in certain regions of the fiber core so that no grating is

\footnotetext{
${ }^{\text {a) }}$ Author to whom correspondence should be addressed. Electronic mail: roberson.oliveira@volvo.com.
}

formed. This mechanism can be compared to the situation where the phase mask is conventionally dithered using piezo elements over the fiber. ${ }^{11}$

Figure 1 illustrates the expected effect. When the fiber is at rest, the UV interferogram produces the usual modulation of the refractive index of the fiber core (Fig. 1(a)). However, when the fiber is flexurally excited (Fig. 1(b)), the core is dithered, thus destroying the fringe interference pattern of the UV light-consequently, no grating is inscribed. This effect can be used to create sampled gratings, fiber cavities, and phase-shifted gratings.

The method of direct writing through a phase mask was employed to inscribe the gratings. The grating writing set-up was modified to make possible the acoustic excitation of the fiber during the process. The fiber is fed through a silica horn, which is attached to a piezo-electric transducer as shown in Fig. 2(a). The resultant flexural acoustic wavelength $\left(\lambda_{s}\right)$, at frequency, $f$, is given by

$$
\lambda_{s}=\sqrt{\frac{\pi a c_{e x t}}{f}},
$$

where $a$ is the fiber radius and $c_{e x t}$ is the extensional acoustic velocity. ${ }^{9}$ For example, when the piezo transducer is driven by $f=113 \mathrm{kHz}$ signal, the achieved flexural wavelength is $\lambda_{s} \sim 3.2 \mathrm{~mm}$, which is usually much shorter than the total grating length used in order for high resolution control along the grating.

The gratings were inscribed in photosensitive step-index fiber without any hydrogen loading. The core of the fiber had a $\mathrm{GeO}_{2}$ doping of $\left[\mathrm{GeO}_{2}\right] \sim 12 \mathrm{~mol}$. \%. A cylindrical lens was used to focus the output beam of a $193 \mathrm{~nm} \mathrm{ArF} \mathrm{laser}$ onto the fiber core and achieve a fluence of $F \sim 300 \mathrm{~mJ} / \mathrm{cm}^{2}$. This radiation was scanned over the phase mask of length $l_{p m}=5 \mathrm{~cm}$, which has a period of $\Lambda_{p m}=1.05 \times 10^{3} \mathrm{~nm}$. The scanning speed was $v=10 \mathrm{~mm} / \mathrm{min}$. In this way, Bragg gratings $5 \mathrm{~cm}$ long were produced, with Bragg wavelength at $\lambda_{B}=1532 \mathrm{~nm}$.

Sampled gratings were originally written by turning ON and OFF, a scanning laser beam either with a fixed amplitude mask or by modulating the laser beam during scanning. ${ }^{2,3}$ To 


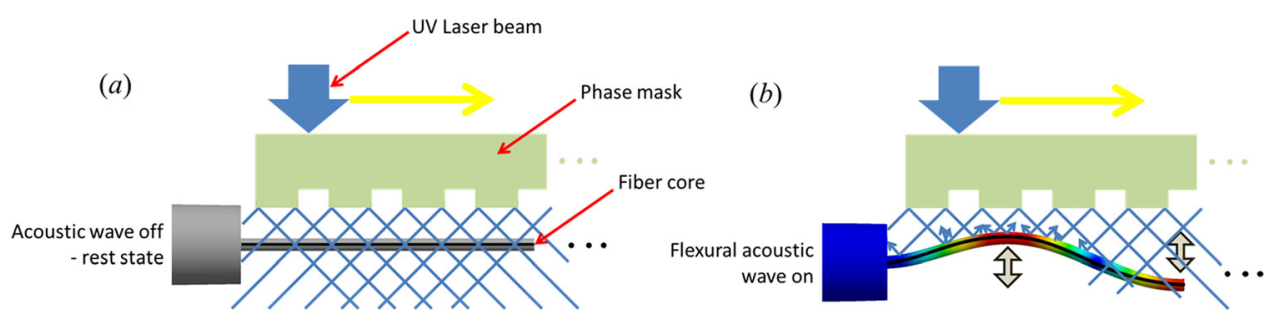

FIG. 1. (Color online) Schematic of the grating writing set-up when (a) no acoustic wave excites the fiber and (b) when a flexural wave excites the fiber.

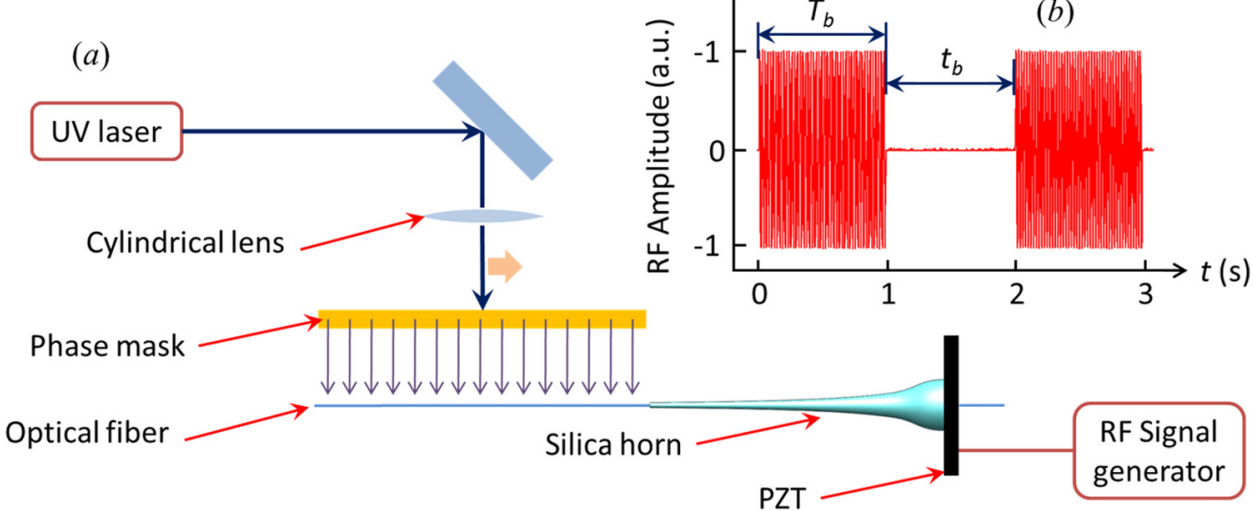

FIG. 2. (Color online) (a) Grating writing set-up showing the modulator positioned in the assembly and (b) burst mode excitation on the PZT. The burst time, $t_{b}$, corresponds to the time between bursts of 100 cycles and $T_{b}$ is the duration of the burst.

achieve the same result by acoustic modulation, as the UV beam is scanned over the phase mask, the flexural wave is periodically turned ON and OFF using the burst function of the signal generator (100 cycles of the acoustic wave were arbitrarily chosen). Fig. 2(b) represents the RF excitation with respect to time, the interval between acoustic bursts is $t_{b}$ and $T_{b}$ is the duration of the burst. When the acoustic wave is present, no grating is written since the frequency is chosen to effectively dither the fiber, whereas when the fiber is at rest, a grating is achieved. It is possible to control the distance between the written gratings by setting the burst parameters (instead of adjusting the modulation of the laser), which is accomplished by means of the acoustic frequency and the UV beam scanning speed.

Fig. 3(a) shows the resultant reflection spectra for different frequencies applied to the modulator. Initially, for comparison purposes, six gratings were inscribed using the conventional technique of modulating the UV writing beam (no acoustic wave exciting the fiber) as it is scanned across the phase mask - the combined spectra are shown in the solid curve of Fig. 3(a). These gratings had a spatial separation of approximately $l_{g} \approx 4.5 \mathrm{~mm}$. Employing now the acoustic technique, the length and number of gratings can be set by controlling the acoustic frequency. For example, considering $f=306 \mathrm{kHz}$, approximately four gratings were written in a $5 \mathrm{~cm}$ long piece of fiber, whereas for $f=75 \mathrm{kHz}$, two gratings were obtained (dotted curves of Fig. 3(a)).

If the frequency is kept constant and the burst time is tuned, this leads to a change in the number of gratings generated and also a change in the length of each grating. Fig. 3(b) shows the resultant reflection spectra when an acoustic frequency of $f=113 \mathrm{kHz}$ excites the fiber using the burst function. The modulation periods were $t_{b}=0.5,1,2$, and $4 \mathrm{~s}$. This gives rise to sampled periods of $\Lambda=1.17,0.57,0.29$, 0.18 , and $0.14 \mathrm{~nm}$, respectively, consistent with that estimated using the standard sampled grating equation described in Ref. 12. It is observed that the sample period is found to decrease when the burst time is increased as shown in Fig. 3(b) - which is expected from the from the Fourier transform of the superperiod. ${ }^{12}$

For the case when the modulation is continuous, the period tends to zero, and a sync-sampled fiber grating ${ }^{13}$ with a phase-shifted spectrum (similar to a phase-shifted Moiré grating $^{14}$ ) is generated, as shown in Fig. 4. The inset scheme of
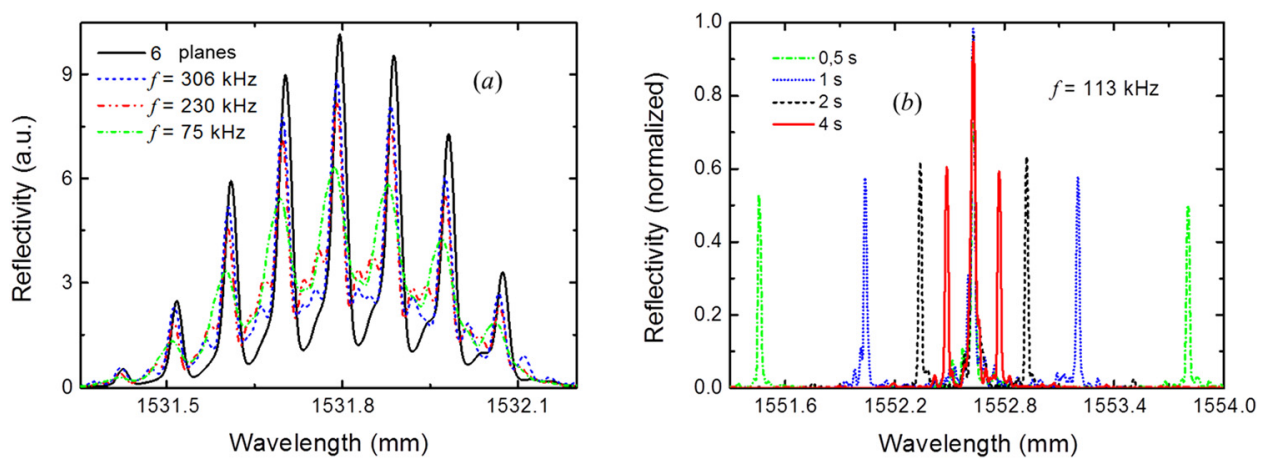

FIG. 3. (Color online) (a) Sampled gratings written using a direct UV beam modulation methodology (solid curve) and by means of acoustic waves at different frequencies. (b) Gratings inscribed by fixing the acoustic frequency and varying the acoustic burst time, $t_{b}$. 


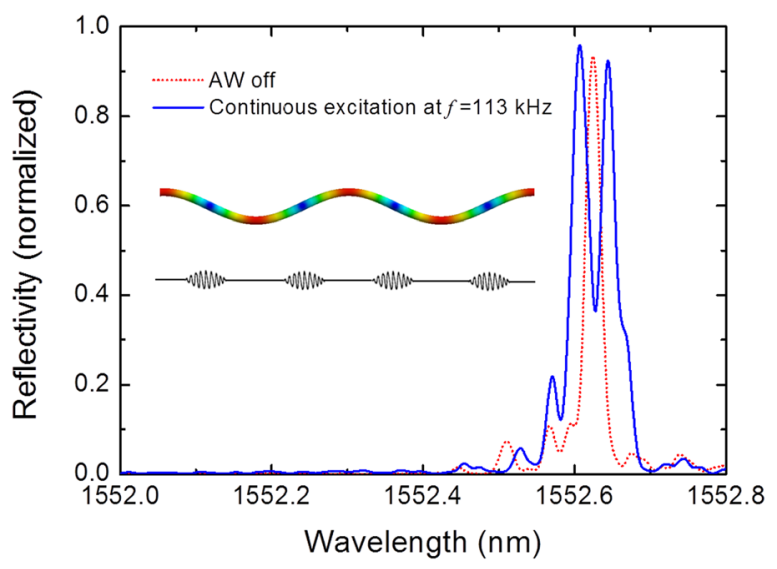

FIG. 4. (Color online) Phase-shifted grating spectrum. Inset: Resultant index modulation shaped by the acoustic wave.

Fig. 4 shows the resultant effective index modulation of the fiber core shaped by the flexural acoustic wave. Note that a different phase mask was used to achieve the results shown in Figs. 3(b) and 4 with length $l_{p m}=2.5 \mathrm{~cm}$ and period that generates a Bragg wavelength of $\lambda_{B}=1552.6 \mathrm{~nm}$.

We have demonstrated the technique of exciting an optical fiber by flexural waves during the process of Bragg grating inscription and have shown that it is possible to create sampling modulation of uniform Bragg gratings as well as phase-shifted complex grating structures, by switching $\mathrm{ON}$ and OFF the acoustic wave during the writing process. The method shows great potential in various applications where precise and fast control of the grating parameters is desired. It is an acoustic equivalent to past processes where the writ- ing tools, such as phase masks, are dithered or the laser beam modulated.

Roberson A. Oliveira thanks the support of Coordenação de Aperfeiçoamento de Pessoal de Ensino Superior (CAPES) - Brazil, by means of the Ph.D. scholarship. The authors also thank the CONTACT (PTDC/EEA-TEL/ 114144/2009) project from the Fundação para a Ciência e Tecnologia (FCT)—Portugal.

${ }^{1}$ K. O. Hill and G. Meltz, J. Lightwave Technol. 15(8), 1263 (1997).

${ }^{2}$ P. Hill, J. Canning, M. G. Sceats and B. J. Eggleton, (1994) (unpublished).

${ }^{3}$ B. J. Eggleton, P. A. Krug, L. Poladian, and F. Ouellete, Electron. Lett. 30, 1620 (1994).

${ }^{4}$ J. Canning and M. G. Sceats, Electron. Lett. 30(16), 1344 (1994).

${ }^{5}$ D. Jiang, X. Chen, Y. Dai, H. Liu, and S. Xie, IEEE Photon. Technol. Lett. 16(12), 2598 (2004).

${ }^{6}$ W. F. Liu, P. St. J. Russell, and L. Dong, Opt. Lett. 22(19), 1515 (1997).

${ }^{7}$ R. A. Oliveira, G. R. C. Posseti, C. A. F. Marques, P. T. Never, Jr., K. Cook, R. C. Kamikawachi, C. Bavastri, M. Muller, J. L. Fabris, J. Canning, R. N. Nogueira, and A. A. P. Pohl, Meas. Sci. Technol. 22, 1228 (2011).

${ }^{8}$ C. A. F. Marques, R. A. Oliveira, A. A. P. Pohl, J. Canning, and R. N. Nogueira, Opt. Commun. 284(5), 1228 (2011).

${ }^{9}$ R. A. Oliveira, K. Cook, J. Canning, and A. A. P. Pohl, Appl. Phys. Lett. 97(1), 041101 (2010).

${ }^{10}$ R. A. Oliveira, P. T. Neves, Jr., J. T. Pereira, J. Canning, and A. A. P. Pohl, Opt. Commun. 283, 1296 (2010).

${ }^{11}$ J. Canning, H.-J. Deyerl, and M. Kristensen, Opt. Commun. 244(1-6), 187 (2005).

${ }^{12}$ V. Jayaraman, Z.-M. Chuang, and L. A. Coldren. IEEE J. Quantum Electron. 29(6), 1824 (1993).

${ }^{13}$ M. Ibsen, M. K. Durkin, M. J. Cole, and R. I. Laming, IEEE Photon. Technol. Lett. 10(6), 842 (1998).

${ }^{14}$ D. C. J. Reid, C. M. Ragdale, I. Bennion, J. Buusm, and W. J. Stewart, Electron Lett. 26(1), 10 (1990). 